\title{
PAYSAGE: PERCEPTION ET PROJET
}

\author{
Author(s) / Auteur(s) : \\ Valerio DI BATTISTA \\ Professore Ordinario di Tecnologia dell'Architettura \\ Politectnico di Milano (POLIMI) \\ dibattistavalerio@libero.it
}

Keywords / Mots-clés :

architecture, paysage, perception, projet

La présente homologation des interventions d'architecture, d'importants complexes, mais aussi des bidonvilles, est fondamentalement égale partout dans le monde (en Chine comme à Dubaï, comme à Rio, etc.) et elle propose la crise de la relation entre les lieux et beaucoup de nos constructions.

Nous avons hérité de la "modernité" la répétitivité du produit. Cela, dans des systèmes d'établissements, dans les villes et les territoires, en rejetant la relation avec l'histoire et le caractère des lieux et a réduit les interactions qui sont indispensables pour la vie des hommes, (comme pour tous les animaux) et des identités qui sont nécessaires pour nous reconnaitre.

Pour surmonter cette crise, il n'y a plus assez de réflexions stylistiques d'architecture, qui sont maintenant facilement subjuguées par l'uniformité des techniques disponibles, des tendances et des processus, des projets et des cycles d'existence de périodes toujours plus courtes. Constatant aussi une approche et des solutions très superficielles dans l'interprétation des caractéristiques stylistiques locales.

C'est une crise culturelle qui regarde notre habitat. Une possibilité pour surmonter cette condition peut uniquement avoir lieu en changeant radicalement le point de vue.

Un besoin de changer est probablement une des raisons du succès que le thème du paysage a dans la culture contemporaine ${ }^{1}$, en particulier avec beaucoup de définitions de la Convention Européenne du Paysage (Firenze, 2000), avec les Recommandations suivantes adoptées par le Comité des Ministres du Conseil de l'Europe (2008) et par celles qui regardent toute formation (Culture du paysage: éducation primaire et secondaire. Recommandation CM/Rec(2014)8 du Comité des Ministres aux Etats membres sur la promotion de la sensibilisation au paysage par l'éducation).

Conceptions qui commencent a trouver des applications dans les plus récents plans de territoires en Europe $^{2}$.

Le paysage nécessite toujours d'envisager tout un système de signes et de significations. La "Convention s'applique à toutes les Parties et porte sur les espaces naturels, ruraux, urbains et périurbains. Elle inclut les espaces terrestres, les eaux intérieures et maritimes. Elle concerne, tant les paysages remarquables, que les paysages du quotidien et les paysages dégradés" (C.E.P. Art. 2).

Cela veut dire que tout le monde est paysage et requiert également une conception nécessairement diachronique qui perçoit l'actualité comme une suite de processus lointains et comme une condition du futur. Et, pour les architectes, cela peut rappeler la définition d'architecture qu'avait donné William Morris en 1881: "L'architecture est l'ensemble des modifications et des altérations sur la surface de la terre, en raison de besoins humains, avec l'exception seulement du désert".

\footnotetext{
${ }^{1}$ Voir bibliographie.

${ }^{2}$ Voir Planification du paysage et des régions: Puglia, Toscana e Catalogna.
} 
Cette conception du paysage comme système complexe de signes et de significations qui inévitablement dénotent tous les écosystèmes naturels (abiotiques et biotiques) et tous ceux des artifices qui dérivent des actions humaines, elle doit surmonter, comme c'est le cas en sciences médicales, la conception dualiste entre l'esprit et le corps. Ce qui signifie dans le paysage surmonter le dualisme naturel - humain et aussi la séparation entre intentionnel et spontané. C'est à dire, pour l'architecture, unifier les approches artistiques et utilitaires, sociales et individuelles, scientifiques et poétiques, dans toutes les différentes échelles. Enfin concevoir l'unité d'un système d'établissement ou les interactions matérielles de sa structure physique (nouvelle et ancienne) ne sont pas séparables des interactions culturelles concernant la population.

Le paradigme du paysage déplace le problème d'une architecture qui se mélange (pour un mimétisme ou contraste) à l'environnement existant et au paysage. Elle devient simplement paysage, partie d'un système de choses qui se modifient toutes, mais avec des temps différents. Elle peut toujours mettre en évidence des intentions individuelles, mais elle peut aussi être concernée par des actions de modifications, et d'interactions spontanées. Cela signifie que c'est le paysage, par sa perception, qui doit motiver et accepter ou refuser toute nouvelle action d'architecture et non l'inverse.

Cela signifie également que le système ne peut pas gouverner toutes les actions individuelles et que par conséquent il va changer grâce à une variété d'intentions qui découlent de raisons différentes et qui se développent avec des moyens et avec des intensités différents. Donc il est configuré comme un résultat chaotique, indéterminé, ouvert et continuel qui accompagne (mais aussi avec des inerties et des conflits) les mêmes processus de changement qui se produisent dans les structures sociales, les activités, les exigences et les comportements de la population.

Le résultat est une coïncidence qui, dans l'analyse et la conception de nos plans, projets, et programmes de gestion, va unifier systèmes observés et systèmes des observateurs. Une coïncidence qui, dans le même système des signes, regarde les résultats qui dérivent simultanément de processus intentionnels ou spontanés. De tout cela dérive d'un schéma chaotique qui va au-delà de toute possibilité de relation déterminante entre la cause et l'effet (la relation usuelle du projet).

La découverte de cette difficulté qui se pose chaque fois que nous pouvons observer ou penser à intervenir dans les systèmes d'établissements et dans leur processus complexes, nécessité l'exploration de nouveaux concepts. La première étape a dépassé les conceptions du projet moderne. Elle s'ouvre de deux façons: l'une a trouvé la liberté créatrice pour concevoir l'architecture comme une grande sculpture, l'autre a redécouvert les problèmes d'interagir avec l'existant. Les deux sont présents aujourd'hui, la première, avec un grand succès médiatique, ouvre la voie à des nouveaux langages, la deuxième, qui concerne les bâtiments, les districts urbains et enfin tout paysage existant, ouvre la voie à de nouvelles méthodes.

Ces méthodes exigent une connaissance et une évaluation approfondie des conditions de l'objet où intervenir avant de définir n'importe quel projet dans le contexte d'une meilleure utilisation des ressources existantes en offrant des économies qui permettent une meilleure distribution et disponibilité.

Ce diagnostic en particulier doit mettre en évidence le potentiel et les valeurs qu'il faudrait confirmer et les problèmes à résoudre. Il remet au projet la tâche de choisir les processus plus efficaces. L'extension de ces principes par les bâtiments et les complexes urbains a commencé à mettre en évidence la présence de nombreux sujets et la réalité des différents processus, qui interagissent les uns avec les autres de façon et à des moments différents.

La considération des problèmes d'environnement ( $c f$. Capra \& Luisi, 2014) met en évidence les interactions entre les systèmes et les processus, à la fois naturels et anthropiques. Mais elle considère les processus anthropiques principalement du point de vue de leurs rétroactions sur les systèmes écologiques (animaux et plantes) et sur les conditions de la sécurité et du bien-être (hygiène et santé) des établissements humains.

En même temps beaucoup de sciences naturelles (en particulier les études de la biologie) et de nombreuses sciences humaines (en particulier les sciences anthropologiques, neurologiques et 
psychologiques) (Bonnes \& Secchiaroli, 2005 ; Mallgrave, 2013, p.17) élaborent des approches qui étendent, de plus en plus, le cadre d'interactions connecté à notre comportement.

Le concept de paysage assumé par le C. E. P.: " 'Paysage' désigne une partie de territoire telle que perçue par les populations, dont le caractère résulte de l'action de facteurs naturels et/ou humains et de leurs interrelations" (C.E.P. Cap. 1, Art.1, A)

"L'objectif de qualité paysagère désigne la formulation par les autorités publiques compétentes, pour un paysage donné, des aspirations des populations en ce qui concerne les caractéristiques paysagères de leur cadre de vie" (C.E.P. Cap. 1, Art.1, C) propose entièrement les conceptions de l'interaction systémique. Il ouvre de nouveaux problèmes et possibilités pour la lecture et les interventions dans les systèmes d'établissements humains (toujours interconnectés à tous les facteurs abiotiques et biotiques de la planète).

Dans cette conception, il est nécessaire d'admettre que la distinction entre les observateurs et les utilisateurs n'existe plus.

En réalité, nous sommes tous des observateurs et tous des utilisateurs. Peut-être, à des moments différents, et, dans les deux cas, avec l'ensemble des bagages de nos motifs et de nos différentes expériences, mais peut-être aussi avec certaines structures organisationnelles de nos pensées et de nos émotions en commun.

Cette hypothèse modifie le sens de nos réflexions vers une méthode qui sait comment se rapprocher de ce cadre complexe d'interactions et de modifications. Elle change nos projets vers des modèles qui se déplacent aussi dans les approches culturelles plus engagées que dans le "goût" de la culture généralisée.

Ce renversement de l'horizon, ce nouveau paradigme que le paysage peut aussi offrir à l'architecture, n'est possible que dans une culture qui est fortement structurée par une vision systémique de notre unité (individuelle et sociale) entre des aspects matériels et immatériels des choses existantes.

L'attention au paysage explicite la nécessité d'examiner les concepts complexes, disponibles aujourd'hui, les systèmes des facteurs abiotiques et biotiques et d'introduire de nouveaux concepts: celui de la variété et de la diversité des acteurs, le rôle des actions involontaires qui dérivent d'interactions aléatoires des actes intentionnels, des organisations structurées et/ou spontanées, enfin des processus instables avec la possibilité qu'émergent" des nouvelles formes d'auto-organisation ${ }^{3}$.

On peut distinguer les sujets actifs de deux grands groupes: celui qui joue de haut en bas (tous les niveaux et plans et programmes institutionnels: tous les experts et les opérateurs du projet) et celui qui joue de bas en haut (toutes les populations à la fois dans des activités productives sur les sols et comme utilisateurs actifs d'espaces). Ces deux sujets offrent des lectures et des évaluations intentionnelles et spontanées avec différents niveaux de conscience et de cohérence. Ces évaluations peuvent motiver des activités qui produisent des résultats de conservation (inaction ou entretien) et des divers résultats de la transformation.

Le paysage dérive de l'ensemble chaotique et aléatoire de ces activités. Toutefois, il met en évidence des formes d'auto-organisation (partielles et locales) de signes et de significations.

Nous avons défini "inintentionnel" le résultat de ces processus, ce terme signifie que les résultats, quelles que soient les intentions, dérivent enfin des additions et des interactions de toutes les activités et les comportements de tous les sujets qui agissent dans nos paysages.

L'inertie et les permanences des oeuvres et en parallèle les changements dans les besoins et dans les activités humaines génèrent des processus ouverts et continus avec différents niveaux d'instabilité.

Il peut être considéré que les réponses à ces instabilités (qui reflètent également les conditions culturelles, économiques et sociales) vont de lancer et de promouvoir l'émergence de niveaux de cohérence entre les signes et les significations.

\footnotetext{
${ }^{3}$ On peut penser qu'au temps passé dans les cultures préindustrielles les architectures et les paysages spontanés montrent des cohérences qui dérivent des cultures matérielles auto-organisées.
} 
Il semble évident, si c'est vrai, que la complexité de nos systèmes d'établissements puisse survenir plus facilement dans les sous-systèmes de paysage sur une échelle locale et non, par exemple, dans les grands paysages des grandes régions.

Cette considération pourrait conduire à confirmer la possibilité de reprendre le problème et le projet de paysage de sa "cellule" constitutive de petite échelle et parvenir, par agrégation, à des configurations, des signes et des significations à des échelles progressivement plus grandes.

Cette hypothèse, en plus de réaffirmer le rôle ascendant des lectures et des gestions à petite échelle, semble faire remarquer un rôle différent pour les niveaux de planification à plus grande échelle (top down).

C'est un rôle qui fournit aux opérateurs les connaissances, et qui facilite les lectures et la prise de décisions locales (participation) en assumant les indications à partir des systèmes d'établissements mineurs qui peuvent, par agrégations successives et itératives, faire prendre des décisions qui pourraient pouvoir offrir des auto-organisations avec cohérence même à plus grande échelle.

Le développement de ces possibilités exige toutefois des conditions convergentes aujourd'hui pas faciles. D'une part le développement, par le biais du processus de formation, d'un raffinement de sensibilité généralisée (et déjà cela exige des modèles culturels progressivement différents de l'actuel) (Morin, 1977); d'autre part le développement et l'essai de conceptions du projet plus articulés et toujours disponibles à différentes formes d'ouverture (back-action) qui permettent des ajustements continus (même avec des fréquences différentes) dans les cycles de vie des diverses présences anthropiques et dans leurs interactions avec les durées très différentes de procédés sur les processus abiotiques et biotiques.

Comme on peut voir la conception C.E.P. du paysage peut impliquer des rôles et des processus très différents de ceux présents. Probablement parce qu'elle pourrait produire, en temps nécessaire, des transformations, des acceptations et des diffusions de ces paradigmes culturels qui ne sont pas évidents.

Les expériences menées aujourd'hui sur les processus de récupération: dans l'éducation, dans la pratique du projet, dans le processus législatif sur les problèmes du paysage à diverses échelles, mettent en évidence l'insuffisance de bon nombre d'outils et de méthodes disponibles aujourd'hui, mais également la grande difficulté de les surmonter, et de ce fait l'exigence d'expérimenter des nouvelles méthodes.

Toutefois, nous sommes convaincus, qu'en surmontant le modèle culturel actuel (ce qui semble être nécessaire par les crises économiques, sociales, productives, écologiques), le paysage, comme structure conceptuelle fortement systémique et avec une forte valence également comme symbole d'utilisation matérielle, puisse donner, même à titre de compensation pour l'extension progressive des symboles virtuels, une possibilité pour améliorer la qualité de nos milieux de vie.

\section{BIBLIOGRAPHIE}

BONNES, M. \& SECCHIAROLI, G. (2005). Psicologia ambientale. Carocci, Roma.

CANTER, D. (1984). Putting situations in their place: foundations for a bridge between social and environmental psychology, in A.Furnham, Social behavior in context, Allyn and Bacon, London.

CAPRA, F. \& LUISI, P.L. (2014). The Systems View of Life, Cambridge University Press.

DEMATTEIS, G. (2007). Paesaggio come "codice genetico". Balletti, F. (a cura di), Sapere tecnico, sapere locale. Conoscenza, identificazione, scenari per il progetto, Alinea, Firenze.

DI BATTISTA, V. (2006). Relazioni lineari e non lineari nel progetto di architettura, in V. Di Battista, G. Giallocosta, G. Minati, Architettura e approccio sistemico, Polimetrica, Milano, p.90.

GUTKIND, E. A. (1958). Architettura e società, Comunità, Milano.

LÜGINBUHL, Y. (2014). La mise en scène du monde. Construction du paysage européen, CNRS edition, Paris.

LYNCH, K. (1960). The Image of the City, Harvard University Press, Cambridge. 
MALLGRAVE, H. F. (2013). Architecture and Embodiment. The Implications of the New Sciences and Humanities for Design, Routledge.

MATURANA, H. \& VARELA, F. (1980). Autopoiesis and cognition, Reidel, Dordrecht.

MORIN, E. (1977). La methode: la nature de la nature, Duseuil, Paris.

MORRIS, W. (1881). The prospect of architecture in civilization, London.

VARELA, F. \& SHEAR, J. (1999). First-person methodologies: what, why, how?, Journal of Consciousness Studies.

C.M./Rec. (2008) 3.

Conseil de l'Europe, Division de la culture et du patrimoine culturel et naturel, Convention Européenne du Paysage, Strasbourg. 
